und Persönlichkeitsentwicklung " der Landesstiftung Baden-Württemberg. Diese Beiträge geben wertvolle Anregungen für eigene Praxisprojekte sowie für weitere Forschungsarbeiten.

Der Band bietet insgesamt einen guten Einblick in laufende Forschungsprojekte zur Genderthematik im Kontext der Diskussion um Medienbildung und Medienkompetenz. Der Titel des Buches „Gender medienkompetent“ mag zunächst irritierend sein, die Lektüre des Buches lohnt sich allerdings unbedingt, zumal die Thematik äußerst aktuell ist.

Renate Luca

\section{Claudia Wegener}

\section{Medien, Aneignung und Identität}

„Stars“ im Alltag jugendlicher Fans

Wiesbaden: VS, 2008. - 419 S.

ISBN 978-3531154428

Fragen zur Sozialisation und Identitätsbildung von Kindern und Jugendlichen wurden von Seiten der Medien- und Kommunikationswissenschaft in den letzten beiden Jahrzehnten schon häufig gestellt. Die Frage nach den medialen Vorbildern, an denen sich Jugendliche orientieren, scheint eines jener Themen, das die Medienpädagogik besonders herauszufordern scheint. Selbst wenn das „Vorbild“ im pädagogischen Stellenwert eingebüßt zu haben scheint, wie Wegener einleitend zu ihrem Thema notiert, „scheint der Vorbild-Status im Rahmen von Leistung und Karriere auch gegenwärtig Relevanz zu besitzen“ (19). Wen wundert das angesichts von Casting-Shows, in denen Vorbildern nachgeeifert wird und die als Format selbst wiederum Vorbildfunktion einnehmen. Angesichts der scheinbar unübersehbaren Vielfalt an „Stars“ und sonstigen Medienfiguren, die für beinahe jede Lebenslage ein „Identifikationsangebot" zu machen scheinen, ist für Wegener der Anteil, den „Medien und Medienpersonen bei der Konstruktion von Identität und der Ausformulierung und Entwicklung von Identitätsentwürfen wie auch Identitätsprojekten haben“, jedoch unklar (49). Diesem Verhältnis in seinen aktuellen Ausprägungen nachzugehen, bildet den Anlass des Buches, das die Ergebnisse des DFG-Projektes „Medienbeziehung und Identitätskonstruktion" zusammenfassend darstellt. Entsprechend umfangreich
(300 Seiten) ist der Untersuchungsteil ausgefallen, dem die Auswertung eines Datensatzes der Studie „Medienhandeln Jugendlicher“ mit 3271 Befragten zugrunde liegt, ferner eine Inhaltsanalyse von Ausgaben der Zeitschrift „Bravo“ vom Herbst 2004 bis Frühsommer 2005 sowie eine Online-Befragung mit 212 Befragten und v. a. Leitfadeninterviews, deren Diskussion den meisten Raum einnimmt (190 Seiten). Behandelt werden Interviews mit acht Jugendlichen aus einem Sample von insgesamt 24 Befragten. Die ausführliche Diskussion der Fallbeispiele behandelt jeweils paarweise ein Mädchen und einen Jungen mit Blick auf die Motive ihrer Vorbildbeziehung und die spezifischen Aneignungsweisen ihrer „medialen Bezugspersonen“ vor dem Hintergrund lebensweltlicher Bezüge. Behandelt werden die Beziehungen zu den „Stars“ Jeanette Biedermann, Britney Spears, Eminem und Robbie Williams.

Der dem umfangreichen Analyseteil vorangestellte theoretische Teil des Buches beschäftigt sich in der Einleitung zunächst mit der Konnotation von Vorbildern, also „Stars“, „Idolen“ und „Helden“. Wegener kommt hier zu dem Schluss, dass diese Begriffe nur jeweils eine spezifische Beziehungsform beschreiben, weswegen sie für die Analyse komplexerer alltagsweltlicher Beziehungen den Begriff der „medialen Bezugsperson“ vorschlägt.

Im zweiten Kapitel wird im Wesentlichen der Forschungsstand des Zusammenhangs von Identität und Medienaneignung reflektiert und darauf aufbauend ein Modell zur „Medienaneignung und Identität" vorgestellt (73), mit dem die Herausforderung, die Identitätskonstruktion im Verhältnis zu „medialen Bezugspersonen“ aufzuschlüsseln, verfolgt wird. Dazu folgt Wegener einem subjektorientierten Konzept von Medienaneignung und interessiert sich dafür, wie Medienthemen in das individuelle Denken und Handeln integriert werden. Identifikation, Identität und Subjektivität erweisen sich damit auch in ihrer Analyse als die Schlüssel, die zur Erklärung in fast jedes Schloss zu passen scheinen, besonders dann, wenn man in subjektzentrierter Perspektive auf die Entwicklung schaut. So stellt das Modell im Wesentlichen auch individuelle Identitätsprojekte in den Vordergrund, welche entlang der Dimensionen Arbeit und Leistung, soziale Beziehung und persönliche Lebensphilosophie als komplexen Identitätsthemen verfolgt werden. 
Die entlang dieser Dimensionen aufschlüsselbaren Identitätsthemen sind für Wegener auch in der Medienaneignung sowie den Motiven der Zuwendung zu medialen Bezugspersonen eine relevante Größe: Identitätsthemen „stellen eine zentrale Frage individueller Konstitution in den Mittelpunkt der Medienaneignung" (72). Mit ihrem subjektorientierten Zugriff reproduziert Wegener in ihren Ergebnissen vielfach jedoch das, was sie an den Identitätstheorien im zweiten Kapitel selbst kritisiert, ihre Unbestimmtheit angesichts medialer Bezüge bzw. Aneignungsweisen. Lässt sich dieser Widerspruch an dieser Stelle aber als Kritikpunkt aufrechterhalten, wenn aus der subjektorientierten Perspektive angesichts der unterstellten Pluralisierung von Lebenswelten kaum ein anderer Weg zu bleiben scheint, als zunächst einmal Identitätsprojekte in ihrem Medienbezug zu durchdringen und darzustellen?

Die relevanten Ergebnisse der Studie liegen v. a. in der Ausleuchtung der Handlungsräume, in denen sich die Sozialisation Jugendlicher aktuell vollzieht. Dabei kommt es Wegener auf die „Transformationen, Verfremdungen und Neugestaltung entsprechender Vorlagen in realen und imaginären Prozessen“ (49) bei der Aneignung medialer Bezugspersonen an. An dieser Stelle findet sich eine Fülle an umfassend aufgearbeiteten und belegten Befunden zu den Formen und Motiven der Zuwendung zu medialen Bezugspersonen, vor den Hintergrund der Bewältigung von Lebensaufgaben, die auch für die Rezeptionsforschung interessante Perspektiven eröffnen. Zweifelsohne ist der Blick auf die Folgen pluraler Lebenswelten zu richten und zweifelsohne finden sich hier Identitätsthemen, bei deren Bewältigung die Medienpädagogik unterstützend eingreifen kann. Dass die "Wahrnehmung von Medienhandeln als subjektiver Aneignung und das Verstehen individueller Deutung“ (386) zum Grundlagenwissen der Medienpädagogik gehören müssen, kann jedoch vor dem Hintergrund des interdisziplinären Anspruchs der Studie nicht darüber hinwegführen, dass grundlegende Fragen zur Subjektkonstitution unbeantwortet bleiben. Diese erscheinen mir erst dann differenzierter behandelbar, wenn eine generalisierende Perspektive angestrebt und nicht wie von Wegener, zurückgewiesen wird.

Udo Göttlich

\section{Dietrich Westphal}

\section{Föderale Privatrundfunkaufsicht im demo- kratischen Verfassungsstaat}

Verwaltungs- und verfassungsrechtliche

Analyse der Kommission zur Ermittlung der

Konzentration im Medienbereich (KEK)

Berlin: Duncker \& Humblot, 2007. - 664 S.

(Schriften zum Öffentlichen Recht; 1072)

ISBN 978-3-428-12318-6

Zugl.: München, Univ., Diss., 2005/2006

Die über 600 Seiten umfassende Dissertation gilt einer Institution, deren Bedeutung rechts- und verwaltungswissenschaftlich längst noch nicht ausgelotet ist. In drei unterschiedlich großen Hauptteilen beleuchtet der Autor die Entstehungsgeschichte der KEK und ihre verfassungs- und europarechtlichen Rahmensetzungen, unternimmt eine verwaltungsrechtliche Beschreibung und Einordnung der KEK und analysiert schließlich die verfassungsrechtlichen Vorgaben. Die Arbeit nimmt dabei weniger die spezifisch medienrechtlichen Bezüge auf und sucht stärker den Anschluss an die Dogmatik des allgemeinen Verwaltungs- und Verfassungsorganisationsrechts.

Die Hälfte der Arbeit (S. 140-450) ist der „Rechtsstruktur der KEK“ gewidmet. Nach einer knappen Zusammenfassung der rechtlichen Grundlagen erörtert der Autor in großer Ausführlichkeit und unter Behandlung aller bekannten Rechtsfragen die Aufgaben und Instrumente. Die aufgeworfenen Fragen werden entlang der einzelnen gesetzlichen Tatbestandsmerkmale und unter umfassender Heranziehung der Literatur und Rechtsprechung wie auch der Entscheidungspraxis der KEK aufgearbeitet.

Das sich anschließende Kapitel zur verwaltungsorganisationsrechtlichen Klassifizierung der KEK (S. 290-360) darf als das interessanteste des Buches gelten. Westphal sucht hierin die KEK in den Kanon der Organisationsformen einzuordnen. Er behandelt die in der Literatur bislang nicht vertieft behandelte Frage nach der „Organ“-Qualität der KEK und begründet mit vielen guten Argumenten, warum sich die Einrichtung diesem Organisationstyp - versteht man ihn klassisch - nicht zuordnen lassen will. Nach richtiger Verneinung des Behördencharakters (und weiterer Zuordnungen) klassifiziert er die KEK als Organisationstyp 